\title{
Models and some properties of Cosserat triangular lattices with chiral microstructure
}

\author{
A. A. Vasiliev ${ }^{\dagger, 1}$, I. S. Pavlov ${ }^{2}$ \\ †alvasiliev@yandex.ru \\ ${ }^{1}$ Tver State University, 35 Sadoviy per., Tver, 170102, Russia \\ ${ }^{2}$ Mechanical Engineering Research Institute of RAS, 85 Belinsky St., Nizhny Novgorod, 603024, Russia
}

\begin{abstract}
Structural, discrete and continual models of the Cosserat triangular lattice with a chiral microstructure are elaborated. The structural model is constructed on the base of particles of finite size with complex spring bonds. With symmetric diagonal bonds, one has the usual Cosserat triangular lattice, while with different diagonal bonds the Cosserat lattice with a chiral microstructure is realized. The choice of different variants enables one to compare the lattices with the ordinary and chiral microstructures and to select the specific properties caused by the chirality. For this purpose, the problems for the hexagonal cell are solved. In the first problem, the behaviour of the cell under a uniform compression (tension) of the cell is investigated. The ordinary lattice is compressed (elongated) in the direction of the force action. The particles of the chiral cell deviate from the direction of the force action during their displacements. The direction of the deviation is different for compressive (tensile) forces, i. e. the chiral lattice responds qualitatively differently to compression and tension. In the second problem, the top and bottom layers of the cell are rigidly compressed (stretched). The middle layer of the ordinary lattice is then stretched (compressed). This is a typical reaction of ordinary materials to tension (compression). The particles displace along the layer. In the chiral lattice, the middle layer is compressed under compression and is stretched under tension of the cell, i.e. chiral cell possesses the auxeticity property. In this case, the middle layer is inclined. The direction of inclination is different for compression or tension. Thus, the chirality effect is manifested. On the basis of the discrete model, equations of the continuum micropolar model are obtained. For symmetric connections, one has equations of the ordinary micropolar theory. The chiral lattice enables one to obtain equations of the chiral micropolar theory and to select the terms of the equations provided by chirality. Using the continuum model, one can find the values of the parameters, for which the second-order derivatives disappear in the rotational equations for the triangular lattice. Thus, it is possible to select a special case of parameters, for which the lattice is described by the equations of the reduced Cosserat theory.
\end{abstract}

Keywords: Cosserat lattice, chirality, structural effects, auxetics.

УДК: 539.3

\section{Модели и некоторые свойства треугольных решеток Коссера с хиральной микроструктурой}

\author{
Васильев А. А. ${ }^{\dagger, 1}$, Павлов И. С. ${ }^{2}$ \\ †alvasiliev@yandex.ru \\ ${ }^{1}$ Тверской государственный университет, Садовый пер., 35, Тверь, 170102, Россия \\ ${ }^{2}$ Институт проблем машиностроения РАН, ул. Белинского, 85, Нижний Новгород, 603024, Россия
}

Разрабатываются структурная, дискретная и континуальная модели треугольной решетки Коссера с хиральной микроструктурой. Структурная модель строится на основе частиц конечного размера со сложными пружинными связями. При симметричных диагональных соединениях имеем обычную треугольную решетку Коссера, а при различных - решётку Коссера с хиральной микроструктурой. Выбор разных вариантов позволяет сравнивать решетки с обычной и хиральной микроструктурой и выделять обусловленные хиральностью особые свойства. Для этого решаются задачи для шестиугольной ячейки. В первой задаче исследуется поведение ячейки при равномерном сжатии (растяжении) ячейки. Обычная решетка сжимается (растягивается) в направлении действия сил. Частицы хираль- 
ной ячейки при смещениях отклоняются от направления действия сил. Направление отклонения различно для сжимающих (растягивающих) сил, т.е. хиральная решетка качественно по-разному реагирует на сжатие и растяжение. Во второй задаче верхний и нижний слой ячейки жестко сжимаются (растягиваются). У обычной решетки средний слой растягивается (сжимается). Это типичная реакция обычных материалов на растяжение (сжатие). Перемещение частиц происходит вдоль слоя. В хиральной решетке средний слой сжимается при сжатии и растягивается при растяжении ячейки, т.е. хиральная ячейка обладает свойством ауксетичности. При этом средний слой наклоняется, причем направление наклона различно при сжатии или растяжении - так проявляется эффект хиральности. На основе дискретной модели получены уравнения континуальной микрополярной модели. Для симметричных соединений имеем уравнения обычной микрополярной теории. Хиральная решетка дает возможность получить уравнения хиральной микрополярной теории и выделить члены уравнений, обусловленные хиральностью. С помощью континуальной модели можно найти значения параметров, при которых вторые производные в уравнениях вращений треугольной решетки пропадают, т. е. выделить особый случай параметров, при которых динамика решетки описывается уравнениями редуцированной теории Коссера.

Ключевые слова: решетка Коссера, хиральность, структурные эффекты, ауксетик.

\section{1. Введение}

В настоящее время повышенный интерес как в плане разработки моделей, так и для технологических применений вызывают метаматериалы, т.е. материалы, обладающие особыми, обусловленными микроструктурой, не характерными для обычных материалов свойствами.

Одним из наиболее широко распространенных примеров метаматериалов являются ауксетики - материалы с отрицательным коэффициентом Пуассона, которые при растяжении в поперечном направлении, в отличие от обычных материалов, увеличивают толщину в продольном направлении, а при сжатии, наоборот, сжимаются. В числе широко обсуждаемых ауксетичских структур были материалы с микроструктурой балочного типа вогнутые сотовые структуры (re-entrant honeycomb structures) [1-5]. В статьях [6,7] предложена структурная модель ауксетика другого типа. Она строилась на основе частиц конечного размера. В статье [8] построена математическая модель таких структур, найдены параметры, при которых решетка такого типа имеет ауксетические свойства и, в частности, показано, что конечность размера частиц и их вращение играют существенную роль для появления ауксетических свойств.

Другим классом представляющих интерес материалов являются материалы и решетки с хиральной микроструктурой. В статьях [9-10] представлены хиральные гексагональные, квадратные, треугольные решётки шарнирно связанных или с соединениями балочного типа частиц конечного размера. В статьях [12,13] построены микрополярные модели таких решеток, а в статье [10] изучаются динамические свойства, в частности, возможности их применения как направленных фононных фильтров частот. Теоретическим и экспериментальным исследованиям хиральных структур, их техническому применению, в частности, для построения инновационных компонент конструкций, посвящена диссертация [11].

В статьях $[14,15]$ нами строятся модели и изучаются свойства квадратных решеток Коссера с хиральной микроструктурой с частицами конечного размера и сложными пружинными связями. В настоящей статье представлена структурная и дискретная модели треугольных решеток, отмечены их интересные свойства, а также строится микрополярная модель, выделяются ее особенности.

\section{2. Структурная и дискретная модели треугольной решетки Коссера с хиральной микроструктурой}

Решетки строятся на основе соединения представленного на Рис. 1 типа. Шестигранные структурные ячейки треугольных решеток с такими соединениями представлены на Рис. 2.

Рассматриваемое соединение носит более общий характер, чем соединение с симметричными связями, которое использовалось для построения моделей решеток в статьях [16-18]. Если жесткости диагональных соединений одинаковы $E_{2}=E_{3}$ имеем симметричное соединение, которое рассматривалось в этих статьях. Несимметричное соединение $E_{2} \neq E_{3}$ позволяет строить хиральные структуры.

Дискретную математическую модель для изучения смещений и вращений можно строить на основе суммирования потенциалов всех пружинных соединений центральной частицы с шестью соседними частицами. Потенциал для соединений пружинного типа частиц конечного размера представлен в статьях $[8,15]$.

Однако, на наш взгляд, представляет интерес построить сначала потенциал сложного соединения двух частиц (Рис. 1). В методе конечных элементов такой подход называется обычно построением супер-элемента. Суммирование потенциалов всех пяти соединений частиц $m$ и $k$ в локальной системе координат после преобразований приводит к потенциалу вида

$$
2 E_{p}=C_{11} \Delta u^{2}+C_{22} \gamma^{2}+C_{33} \Delta \varphi^{2}+C_{12} \gamma \Delta u
$$

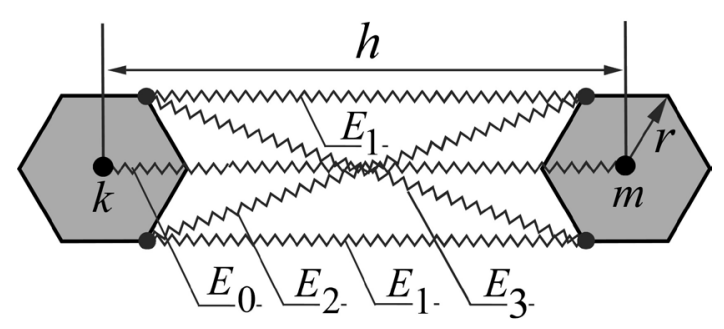

Рис. 1. Сложное пружинное соединение частиц конечного размера.

Fig. 1. Complex spring bond of particles of finite size. 


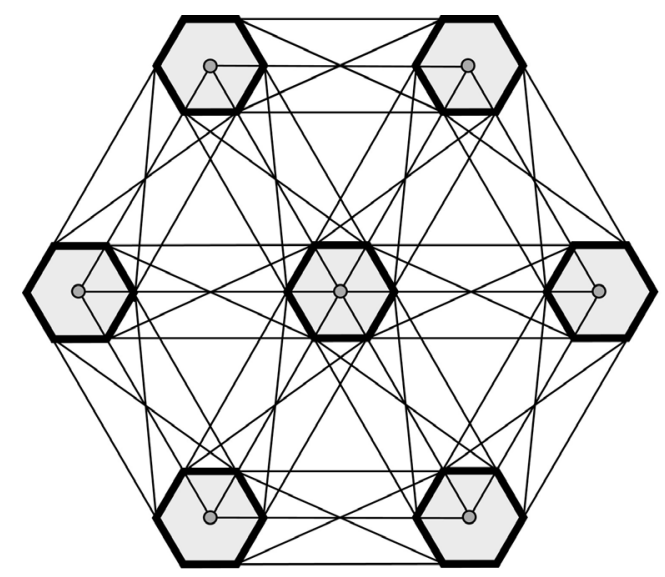

a

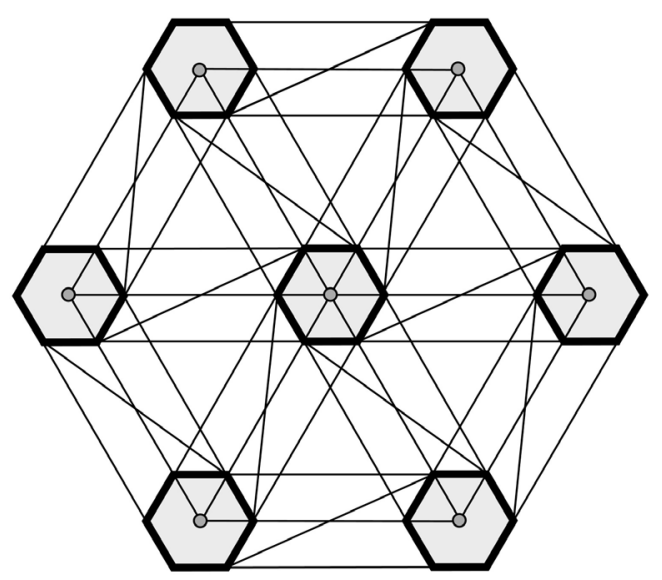

b

Рис. 2. Шестигранные ячейки с симметричным (a) и хиральным (b) соединением частиц.

Fig. 2. Hexagonal cells with a symmetrical (a) and chiral (b) bond of particles.

где использованы обозначения:

$$
\begin{gathered}
\Delta u=u_{m}-u_{k}, \gamma=v_{m}-v_{k}-\frac{1}{2} h\left(\varphi_{m}+\varphi_{k}\right), \\
\Delta \varphi=\varphi_{m}-\varphi_{k}, C_{11}=E_{0}+2 E_{1}+\frac{(h-r)^{2}}{d^{2}}\left(E_{2}+E_{3}\right), \\
C_{22}=3 \frac{r^{2}}{d^{2}}\left(E_{2}+E_{3}\right), C_{33}=\frac{3}{2} r^{2} E_{1}, \\
C_{12}=2 \sqrt{3} \frac{r(h-r)}{d^{2}}\left(E_{2}-E_{3}\right)
\end{gathered}
$$

$u, v-$ перемещения в горизонтальном и вертикальном направлениях, $\varphi-$ угол вращения частиц, $r-$ радиус частиц, $h-$ расстояние между частицами, $d=\sqrt{h^{2}-2 r h+4 r^{2}}$ - длина диагональных соединений.

В случае $E_{2}=E_{3}$ в потенциале (1) коэффициент $C_{12}=0$, а в длинноволновом континуальном приближении он аналогичен потенциалу простейшего варианта одномерной микрополярной теории упругости. Выражения коэффициентов $C_{11}, C_{22}, C_{33}$ потенциала (1) через параметры балочного и сложного симметричного пружинного соединения получены в статье [19]. Для соединения хирального типа $\left(E_{2} \neq E_{3}\right)$ коэффициент $C_{12} \neq 0$ и соответствующая дополнительная компонента в потенциале (1) появляется.

Дискретные уравнения строятся с использованием стандартного алгоритма метода конечных элементов в пакетах символьной математики, например, Maple. Получаемые уравнения громоздки, поэтому мы их не приводим.

\section{3. Особые свойства ячеек с хиральной микроструктурой}

Развитие механики метаматериалов связано с построением структур, обладающих особыми свойствами, анализом таких свойств, поиском путей их практических применений. В настоящем разделе мы отметим два таких свойства.

\section{1. Несимметрия реакций на воздействия}

Численно исследовалась деформация шестигранных ячеек под действием равномерного симметричного (часто называют гидростатического) растяжения (сжатия). Расчет проводился для ячейки с симметричными связям $\left(E_{2}=E_{3}\right)$ и ячейки с предельным вариантом хиральных связей $\left(E_{2} \neq E_{3}\right)$, когда одно из диагональных соединений отсутствует $E_{2} \neq 0, E_{3}=0$. Это позволяет выявить особенности деформирования хиральных структур.

Результат для ячейки с симметричными связями Рис. 2 а для гидростатического растяжения представлен на Рис. 3 а. На рисунках серым цветом выделена исходная шестигранная ячейка, показаны соединения центров частиц ячейки в исходном состоянии. Ячейка после деформации не заштрихована. Видно, что в симметричной ячейке частицы смещаются в направлении действия сил, а центральная частица не смещается. Это очевидно и из физических соображений.

Результат для ячейки с хиральной микроструктурой Рис. 2 b представлен на Рис. 3 b и Рис. 3 с для растяжения и сжатия соответственно. В хиральной структуре частицы смещаются не вдоль нагрузок, отклоняясь от направления действия сил. Другой более интересный результат, который можно отметить, состоит в том, что закручивание (отклонение) происходит в разных направлениях в зависимости от того, действуют сжимающие или растягивающие силы, то есть система в этом смысле качественно различно реагирует на сжимающие и растягивающие нагрузки.

Анализ показывает, что в рассмотренной задаче есть только четыре независимые переменные: смещения $u, v$ и вращение $\varphi$ одной из частиц периметра ячейки и поворот центральной частицы. Найдя эти величины, можно описать деформирование ячейки.

\section{2. Ауксетические свойства}

Численно исследовалось деформирование шестигранной ячейки под действием жесткого $\left(u=0, v^{*} \neq 0, \varphi=0\right)$ растяжения (сжатия) частиц верхней и нижней граней. 
Для выявления особенности поведения ячейки с хиральной микроструктурой расчет проводился для ячейки с симметричными связями $\left(E_{2}=E_{3}\right)$ и ячейки с предельным вариантом хиральных связей, $E_{2} \neq 0, E_{3}=0$. При расчетах рассматривались ячейки с введенными соединениями одинаковой жесткости и соотношением геометрических параметров $r / d=0.2$.

Результат для ячейки с симметричными связями представлен на Рис. 2 а. Средний слой симметрично без вертикальных смещений сжимается (Рис. 4 a) при растяжении частиц верхней и нижней граней. При сжатии слой растягивается. Это соответствует поведению обычного неауксетического материала.

Результат для ячейки с хиральной микроструктурой (Рис. 2 b) представлен на Рис. $4 \mathrm{~b}$ и Рис. 4 с для жесткого растяжения и сжатия ячейки соответственно. При растя- жении частиц верхней и нижней граней средний слой расширяется (Рис. 4b), при сжатии - сжимается (Рис. 4c). Таким образом, хиральная решетка обладает ауксетическими свойствами. Взятое с обратным знаком отношение относительного изменения толщины ячейки по оси $O_{x}$ к относительному изменению толщины в направлении растяжения (сжатия) вдоль оси $O_{y}$ равно -0.988 , что близко к предельному для случая плоского континуального материала значению коэффициента Пуассона $v=-\varepsilon_{x} / \varepsilon_{y}=-1$. Крайние частицы центрального слоя ячейки смещаются в поперечном направлении относительно центральной оси, но в разных направлениях при растяжении и сжатии.

Анализ результатов показывает, что в рассмотренной задаче четыре независимые переменные: смещения $u, v$ и вращение $\varphi$ одной из частиц периметра и поворот центральной частицы.
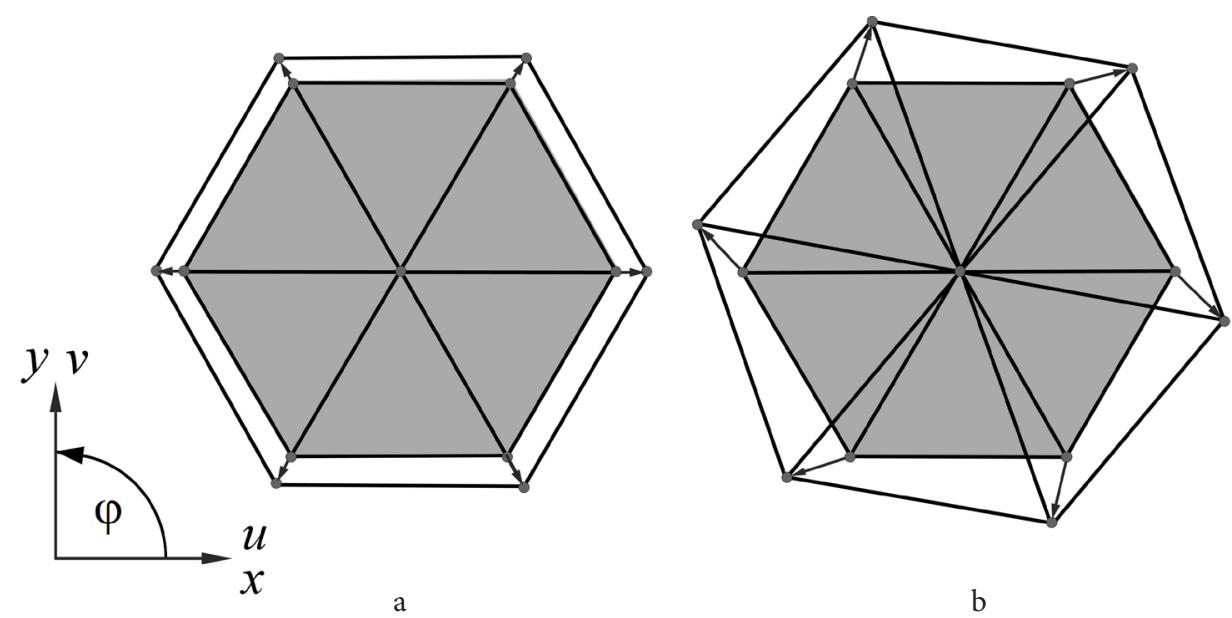

$\mathrm{b}$

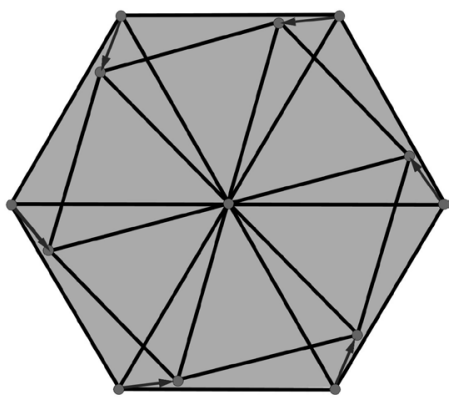

c

Рис. 3. Деформация шестигранной ячейки с симметричными связями под действием растягивающих сил (а). Деформация ячейки с хиральной микроструктурой под действием растягивающих (b) и сжимающих (c) сил

Fig. 3. Deformation of a hexagonal cell with symmetrical bonds under the action of tensile forces (a). Deformation of a cell with a chiral microstructure under the action of tensile (b) and compressive (c) forces

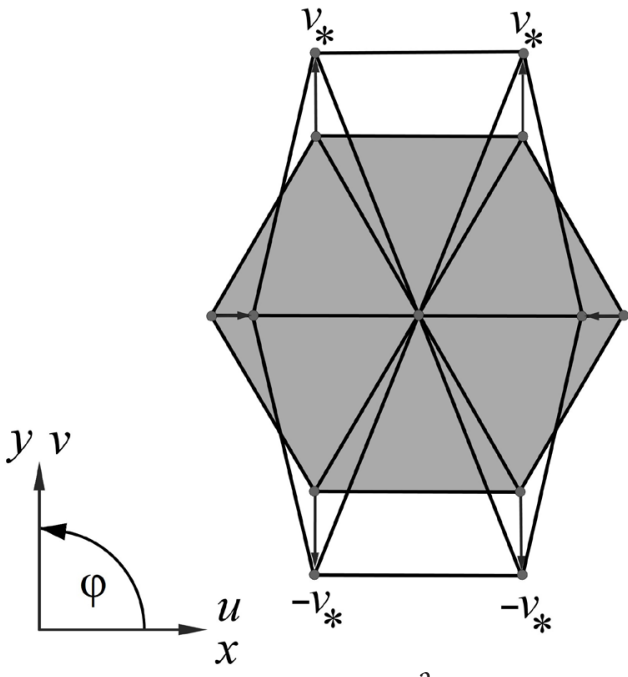

a

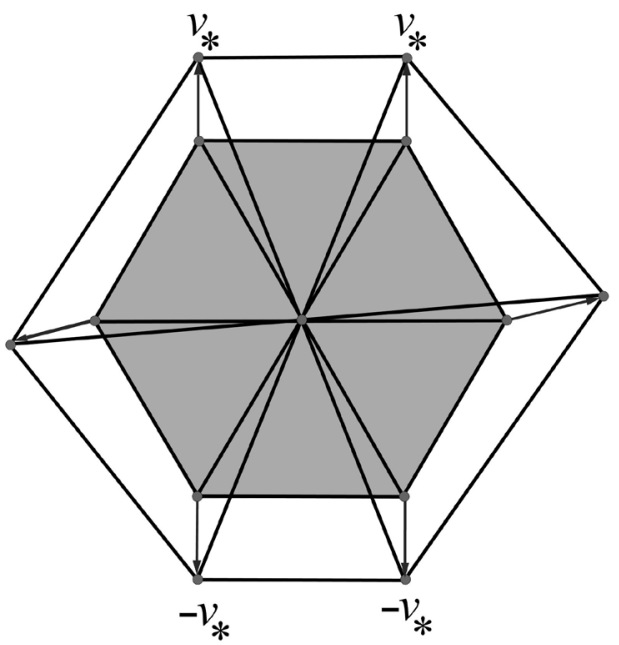

$\mathrm{b}$

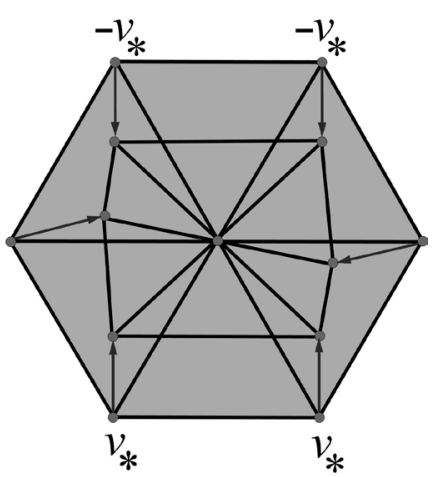

Рис. 4. Деформация шестигранной ячейки с симметричными связями при растяжении нижнего и верхнего слоев (а). Деформация ячейки с хиральной микроструктурой при растяжении (b) и сжатии (c) верхнего и нижнего слоев

Fig. 4. Deformation of a hexagonal cell with symmetrical bonds, when the lower and upper layers are stretched (a). Deformation of a cell with a chiral microstructure under tension (b) and compression (c) of the upper and lower layers 


\section{4. Микрополярная модель треугольной решетки Коссера с хиральной микроструктурой}

Длинноволновые континуальные уравнения решетки получаются из дискретных уравнений разложением в ряд Тейлора компонент перемещений и вращения частиц ячейки. Поскольку такие уравнения представляют интерес, приведем их:

$$
\begin{aligned}
\rho u_{t t}= & C_{2}\left(\frac{1}{2} v_{x x}-u_{x y}-\frac{1}{2} v_{y y}\right)+C_{3} u_{x x}+C_{6} v_{x y}+ \\
& +C_{4} u_{y y}-C_{2} \varphi_{x}-\frac{1}{2} C_{1} \varphi_{y}, \\
\rho v_{t t}= & C_{2}\left(\frac{1}{2} u_{x x}+v_{x y}-\frac{1}{2} u_{y y}\right)+C_{4} v_{x x}+C_{6} u_{x y}+ \\
& +C_{3} v_{y y}-C_{2} \varphi_{y}+\frac{1}{2} C_{1} \varphi_{x}, \\
j \varphi_{t t}= & C_{5}\left(\varphi_{x x}+\varphi_{y y}\right)+\frac{1}{2} C_{1}\left(u_{y}-v_{x}+2 \varphi\right)+ \\
& +C_{2}\left(u_{x}+v_{y}\right),
\end{aligned}
$$

где введены обозначения $\rho=2 \sqrt{3} M / 9 h^{2}$, $j=2 \sqrt{3} J / 9 h^{2}, M-$ масса частиц, $J-$ их момент инерции, а также обозначения для коэффициентов:

$$
\begin{aligned}
& C_{1}=-4 \sqrt{3} c_{2}, \quad C_{2}=2 c_{3}, \quad C_{3}=\frac{\sqrt{3}}{4}\left(c_{0}-4 c_{2}\right), \\
& C_{4}=\frac{1}{12} \sqrt{3}\left(c_{0}+12 c_{2}\right), C_{5}=\frac{\sqrt{3}}{12} h^{2}\left(c_{1}-6 c_{2}\right), \\
& C_{6}=\frac{1}{12} \sqrt{3}\left(c_{0}-12 c_{2}\right) .
\end{aligned}
$$

Коэффициенты $C_{k}$ выражаются через коэффициенты жесткости упругих соединений:

$$
\begin{aligned}
& c_{0}=E_{0}+2 E_{1}+E_{2}+E_{3}, c_{1}=6 \frac{r^{2}}{h^{2}} E_{1}, \\
& c_{2}=\frac{1}{2} \frac{r^{2}}{d^{2}}\left(E_{2}+E_{3}\right), c_{3}=\frac{1}{2} \frac{r(h-r)}{d^{2}}\left(E_{2}-E_{3}\right) .
\end{aligned}
$$

Относительно полученных уравнений сделаем несколько замечаний.

При формировании теорий обобщенной механики, как и моделей классической механики деформируемого твердого тела, интересны два подхода: феноменологический и структурный. Структурный подход обеспечивает предметное понимание формируемой теории, возможность ее практической реализации, дает выражение макропараметров материала через микропараметры, что, в частности, дает возможность формировать материалы с заданными свойствами или наоборот, зная макропараметры, исследовать микроструктуру [20,21,16-18].
Уравнения хиральной микрополярной теории получены нами на основе структурного подхода. В случае симметричных соединений $E_{2}=E_{3}$ параметр $c_{3}$ равен нулю и соответственно равен нулю коэффициент $C_{2}$. Соответствующие составляющие в уравнениях (2) отсутствуют. В этом случае получаем уравнения классической микрополярной теории. Случай уравнений с коэффициентом $C_{2} \neq 0$ соответствует варианту хиральной микрополярной теории, описывающему треугольную решетку. Соответственно треугольная решетка является вариантом реализуемой структуры, соответствующей этим уравнениям.

Заметим, что при $c_{1}=6 c_{2}$ коэффициент $C_{5}$ равен нулю и вторые производные в уравнения вращений отсутствуют. Уравнения в таком случае называют уравнениями редуцированной теории Коссера. В статье [22] такие уравнения вводились на основе феноменологического подхода, решались задачи динамики и изучались свойства сред, соответствующих такому варианту теории Коссера. В данной статье предложена структурная модель решетки, которая соответствует уравнениям хиральной континуальной редуцированной теории Коссера и может быть реализована практически.

\section{5. Заключение}

В статье введена структурная модель треугольной решетки Коссера с хиральной микроструктурой. На основе исследования ячейки, показано, что она обладает интересным свойством несимметричной реакции на воздействия, различной реакцией на растяжение/сжатия, свойством ауксетичности. Построена микрополярная модель, выявлены параметры, соответствующие случаю редуцированной модели Коссера.

Рассматриваемая решетка строится с использованием частиц конечного размера и сложных связей пружинного типа. В отличии от решеток с соединениями балочного типа, которые в прикладном плане в большей мере ориентированы на применение в технических системах, решетки частиц конечного размера ориентированы на моделирование материалов с микроструктурой.

Отметим несколько направлений дальнейших исследований, для которых настоящая статья может являться базовой.

Исследование свойств метаматериалов часто аналитически проводят для бесконечных решеток, а для многоячеечных конечных структур - на основе натурных или вычислительных экспериментов. Для выявления особых свойств, на наш взгляд, представляет интерес проведенное в этой статье исследование простейших ячеечных структур. Такой анализ, по-видимому, недооценён. В разделе 3 статьи мы приводим некоторые интересные свойства шестигранных ячеек. Отметим, что такие исследования помогают также выделить симметрии деформирования и сократить число неизвестных переменных для дальнейших аналитических исследований.

В статье не рассмотрены динамические свойства решеток. Их исследование, в частности, построение на основе представленных в статье хиральных структур направленных фильтров частот $[3,11]$ и решение нели- 
нейных задач динамики [23] для решеток с учетом размера частиц требует отдельной статьи. Исследование особых динамических свойств сред, описываемых редуцированными уравнениями Коссера (раздел 4), обобщение результатов статей для линейного [22] и нелинейного [24] варианта таких сред также, на наш взгляд, также представляет интерес.

Благодарности/Acknowledgements. Исследование проводилось при финансовой поддержке Российского фонда фундаментальных исследований (проекты №16-08-00971-a, №18-29-10073 мк) и Министерства образования и науки Российской Федерации в рамках базовой части государственного задания (проект №9.7446.2017/БЧ)./The research was carried out under the financial support of the Russian Foundation for Basic Research (projects N 16-08-00971-a, №18-29-10073 MK) and the Ministry of Education and Science of Russian Federation within the framework of the basic part of State Work for scientific activity (Work No. 9.7446.2017/8.9).

\section{Литература}

1. A. G. Kolpakov. J. Appl. Math. Mech. 49 (6), 739 (1985). DOI: 10.1016/0021-8928(85)90011-5

2. D. Prall, R. S. Lakes. Int. J. of Mech. Sci. 39, 305 (1997). DOI: 10.1016/S0020-7403(96)00025-2

3. M. Ruzzene, F. Scarpa. Physica Status Solidi (b). 242 (3), 665 (2005). DOI: 10.1002/pssb.200460385

4. R.V. Goldstein, D.S. Lisovenko, A.V. Chentsov, S. Yu. Lavrentyev. Letters on Materials. 7 (2), 81 (2017). DOI: 10.22226/2410-3535-2017-2-81-84

5. R.V. Goldstein, D.S. Lisovenko, A.V. Chentsov, S. Yu. Lavrentyev. Letters on Materials. 7 (4), 355 (2017). DOI: 10.22226/2410-3535-2017-4-355-358

6. Y. Ishibashi, M. Iwata. J. Phys. Soc. Jpn. 69 (8), 2702 (2000). DOI: 10.1143/JPSJ.69.2702

7. J. N.Grima,K. E.Evans. JournalofMaterialsScienceLetters. 19 (17), 1563 (2000). DOI: 10.1023/A:1006781224002

8. A.A. Vasiliev, S.V. Dmitriev, Y. Ishibashi, T. Shigenari. Phys. Rev. B. 65, 094101 (2002). DOI: 10.1103/PhysRevB.65.094101

9. J. N. Grima, R. Gatt, P.-S. Farrugia. Physica Status Solidi (b).
245 (3), 511 (2008). DOI: 10.1002/pssb.200777704

10. A. Spadoni, M. Ruzzene, S. Gonella, F. Scarpa. Wave Motion. 46, 435 (2009). DOI: 10.1016/j.wavemoti.2009.04.002

11. A. Spadoni. Application of chiral cellular materials for the design of innovative components: $\mathrm{PhD}$ thesis. Atlanta. (2007) $230 \mathrm{p}$.

12. A. Spadoni, M. Ruzzene. J. Mech. Phys. Solids. 60, 156 (2012) DOI: 10.1016/j.jmps.2011.09.012

13. X. N. Liu, G. L. Huang, G. K. Hu. J. Mech. Phys. Solids. 60, 1907 (2012). DOI: 10.1016/j.jmps.2012.06.008

14. A. A. Vasiliev. Letters on Materials. 3, 248 (2013). (in Russian) [А.А. Васильев. Письма о материалах. 3, 248 (2013).] DOI: 10.22226/2410-3535-2013-3-248-251

15. A.A. Vasiliev, S. V. Dmitriev, I.S. Pavlov. Advanced materials. 12, 87 (2011). (in Russian) [А.А. Васильев, С. В. Дмитриев, И.С. Павлов. Перспективные материалы. 12, 87 (2011).]

16. A. I. Potapov, I. S. Pavlov, S. A. Lisina. Acoustical Physics. 56 (4), 588 (2010). DOI: 10.1134/S1063771010060175

17. I.S. Pavlov, A.A. Vasiliev, A.V. Porubov. Journal of Sound and Vibration. 384, 163 (2016). DOI: $10.1016 /$ j.jsv.2016.08.012

18. V.I. Erofeev, I.S. Pavlov, A.A. Vasiliev, A.V. Porubov. Advanced Structured Materials. 90, 101 (2018). DOI: 10.1007/978-3-319-77504-3_5

19. A.A. Vasiliev, A.E. Miroshnichenko, M. Ruzzene. Mechanics Research Communications. 37 (2), 225 (2010). DOI: 10.1016/j.mechrescom.2009.11.010

20. V.I. Erofeev, I. S. Pavlov. J. Appl. Mech. Tech. Phys. 56 (6), 1015 (2015). DOI: 10.1134/S0021894415060115

21. R. V. Goldstein, V.A. Gorodtsov, D.S. Lisovenko. Letters on Materials. 1 (3), 127 (2011). (in Russian) [Р. В. Гольдштейн, В.А. Городцов, Д.С. Лисовенко. $\begin{array}{llllll}\text { Письма о материалах. } 1 & \text { (3), } 127 & \text { (2011).] }\end{array}$ DOI: 10.22226/2410-3535-2011-3-127-132

22. E. F. Grekova, M. A. Kulesh, G. C. Herman. Bull. Seismol. Soc. Am. 99 (2B), 1423 (2009). DOI: 10.1785/0120080154

23. A.V. Porubov, I.E. Berinskii. Mathematics and Mechanics of Solids. 21 (1), 94 (2016). DOI: $10.1177 / 1081286515577040$

24. A.A. Vasiliev, A.E. Miroshnichenko. Letters on materials. 7 (4), 388 (2017). DOI: 10.22226/2410-3535-2017-4-388-392 